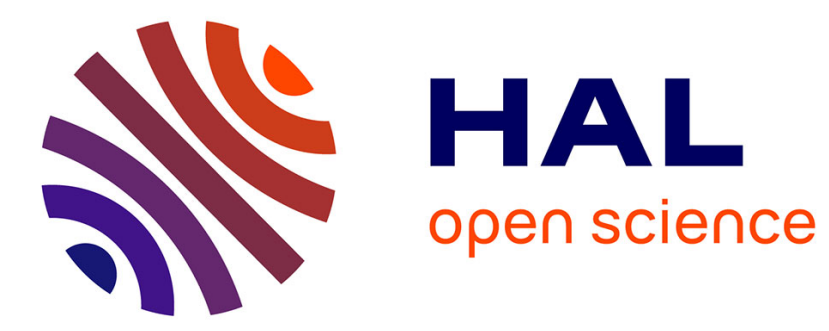

\title{
Laser-assisted evaporative cooling of anions
}

G Cerchiari, P Yzombard, A Kellerbauer

\section{- To cite this version:}

G Cerchiari, P Yzombard, A Kellerbauer. Laser-assisted evaporative cooling of anions. Physical Review Letters, 2020. hal-03000318

\section{HAL Id: hal-03000318 https://hal.science/hal-03000318}

Submitted on 11 Nov 2020

HAL is a multi-disciplinary open access archive for the deposit and dissemination of scientific research documents, whether they are published or not. The documents may come from teaching and research institutions in France or abroad, or from public or private research centers.
L'archive ouverte pluridisciplinaire HAL, est destinée au dépôt et à la diffusion de documents scientifiques de niveau recherche, publiés ou non, émanant des établissements d'enseignement et de recherche français ou étrangers, des laboratoires publics ou privés. 


\title{
Laser-assisted evaporative cooling of anions
}

\author{
G. Cerchiari, P. Yzombard, and A. Kellerbauer* \\ Max Planck Institute for Nuclear Physics, Saupfercheckweg 1, 69117 Heidelberg, Germany
}

(Dated: May 12, 2020)

\begin{abstract}
We report the first cooling of atomic anions by laser radiation. $\mathrm{O}^{-}$ions confined in a linear Paul trap were cooled by selectively photodetaching the hottest particles. For this purpose, anions with the highest total energy were illuminated with a $532 \mathrm{~nm}$ laser at their maximal radial excursion. Using laser-particle interaction, we realized a both colder and denser ion cloud, achieving a more than threefold temperature reduction from $1.15 \mathrm{eV}$ to $0.33 \mathrm{eV}$. Compared with the interaction with a dilute buffer gas, the energy-selective addressing and removal of anions resulted in lower final temperatures, yet acted 10 times faster and preserved twice as large a fraction of ions in the final state. An ensemble of cold negative ions affords the ability to sympathetically cool any other negative ions species, enabling or facilitating a broad range of fundamental studies from interstellar chemistry to antimatter gravity. The technique can be extended to any negative ion species that can be neutralized via photodetachment.
\end{abstract}

5 Laser cooling, which reduces the kinetic energy of neutral 6 or charged particles, is one of the most important experimental 7 tools in atomic physics. It allows the cooling of particle beams 8 or ensembles to temperatures below that of the surrounding 9 apparatus, well below $1 \mathrm{~K}$. Ultracold temperatures are a pre10 requisite for many groundbreaking applications, such as ultra11 precise clocks [1], quantum information [2], quantum compu12 tation [3], or the study of matter waves with Bose-Einstein ${ }_{13}$ condensates $[4,5]$. In the case of charged particles, laser cool14 ing enables the formation of Coulomb crystals, regularly or15 dered structures that form when the electrostatic interaction 16 prevails over thermal motion [6]. One interesting application 17 of laser-cooled ultracold ions is the sympathetic cooling of ${ }_{18}$ another particle species. For instance, highly charged ${ }^{40} \mathrm{Ar}^{13+}$ 19 ions have been cooled into an ordered crystalline structure 20 suitable for precision spectroscopy by interacting with $\mathrm{Be}^{+}$ ${ }_{21}$ ions at $\mathrm{mK}$ temperatures [7]. Other state-of-the-art experi22 ments have combined trapped ions with a magneto-optical ${ }_{23}$ trap in such a way that the laser-cooled neutral atoms con24 stitute a buffer medium which cools the trapped ions by colli25 sions $[8,9]$.

26 In negative ions, the electronic binding is characterized by 27 a short-range potential instead of the infinite-range Coulomb 28 potential. Because of their fragile nature, the laser cooling of 29 negative ions has not yet been achieved. Laser-cooled anions ${ }_{30}$ would open the field of ultra-precision studies to a new kind ${ }_{31}$ of system. Furthermore, they could be used to sympathetically 32 cool any other negatively charged particles [10]. In the case of зз antimatter, a neutral buffer medium cannot be used for cool34 ing due to the risk of annihilation, whereas the repelling elec35 tric charge of anions would prevent them from annihilating ${ }_{36}$ with antiprotons. In this way, a sample of ultracold antipro37 tons could be prepared by collisions with laser-cooled nega38 tive ions. Antihydrogen subsequently formed with these an39 tiprotons would have almost the same low temperature due to 40 the large mass ratio of antiproton to positron. This antihydro${ }_{41}$ gen production technique would open the way for a variety of 42 high-precision antimatter experiments. For instance, a beam 43 of antihydrogen at $\approx 100 \mathrm{mK}$ is a prerequisite for an antimat${ }_{44}$ ter gravity measurement to test the weak equivalence principle
45 using a moiré deflectometer [11].

46 Due to the weak binding of the valence electron, only very 47 few atomic $[12,13]$ or molecular $[14,15]$ anions are amenable 48 to laser cooling. Recently, $\mathrm{La}^{-}$has been identified as the most 49 promising atomic-anion candidate to date $[16,17]$. The po50 tential Doppler cooling transition has been fully characterized $51[18,19]$. The existence of a dark state, as well as photode52 tachment of the excited $\mathrm{La}^{-}$by the cooling laser, impose an ${ }_{53}$ upper limit of $100 \mathrm{~K}$ on the initial temperature. Starting from 54 that temperature, an ensemble of $\mathrm{La}^{-}$ions could be cooled to ${ }_{55}$ the Doppler temperature $T_{D}=0.17 \mu \mathrm{K}$ within a few seconds. ${ }_{56}$ When ions are loaded into an ion trap, they typically have 57 temperatures of several ten thousand K. In order to precool 58 them to the starting temperature for Doppler cooling, we have 59 implemented a scheme based on the selective laser photode60 tachment of the hottest trapped particles, as suggested almost 61 thirty years ago [20]. This technique is a variant of evapora62 tive cooling, which is a well-established cooling mechanism 63 for atoms [21] and, to a lesser extent, ions [22]. Evaporative 64 cooling by photodetachment is only applicable to (atomic or 65 molecular) anions, because neither cations nor negative sub${ }_{66}$ atomic particles can be removed from an ion trap by photode67 tachment. In this Letter we report the successful cooling of ${ }_{68} \mathrm{O}^{-}$ions with this technique, employing geometrical selection. ${ }_{69}$ This is the first demonstration of the direct cooling of a nega70 tive ion species using optical radiation.

The experiments reported here were carried out with $\mathrm{O}^{-}$ 72 ions (electron affinity $E_{A}=1.4611136(9) \mathrm{eV}$ [23], using the 73 most recent conversion factor from $\mathrm{m}^{-1}$ to $\mathrm{eV}$ [24]) confined 74 in a linear Paul trap. The trap, the trapping method, as well 75 as the detection technique are described in detail in Ref. [25]. ${ }_{76}$ Briefly, the linear trap is composed of four cylindrical parallel 78 electrodes (rods). A cross-sectional view is shown in Fig. 1. ${ }_{79}$ The rods have a diameter of $14.8 \mathrm{~mm}$ and a distance between so the trap axis and the inner electrode surface of $r_{0}=7 \mathrm{~mm}$. ${ }_{81}$ A radiofrequency (RF) signal is applied to one pair of op82 posing rods, whereas the signal on the second pair of rods ${ }_{83}$ has a phase shift of $\pi$. The applied RF has a frequency of ${ }_{84} \Omega_{R F} /(2 \pi)=1.938 \mathrm{MHz}$ and an amplitude (peak-to-peak) of ${ }_{85} V_{p p}=225(20) \mathrm{V}$. The amplitude was chosen for optimal ion 


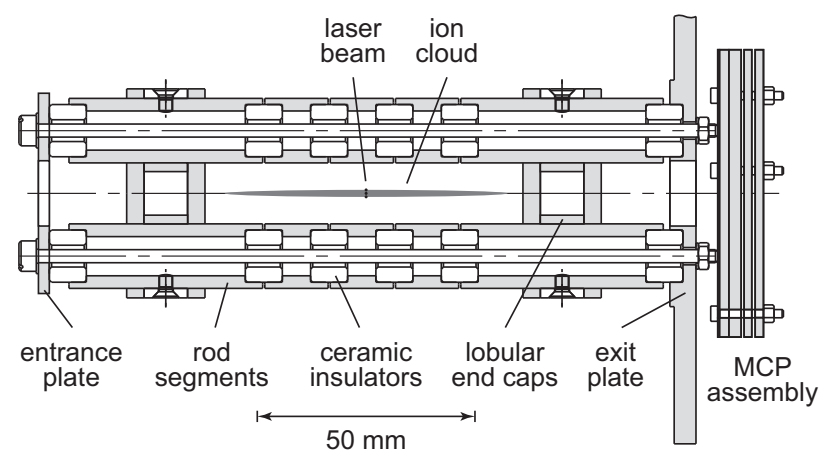

FIG. 1. Schematic drawing of the linear Paul trap. Ions enter the trap from the left and exit towards the MCP detector to the right.

86 loading. Additional electrodes (lobe-shaped end caps) pro${ }_{87}$ vide axial ion confinement in a region of $65(5) \mathrm{mm}$ length. ${ }_{88}$ Photodetachment of the anions is achieved by the light of a $89532 \mathrm{~nm}$ laser $(h v=2.33 \mathrm{eV})$ focused on the trap region.

90 The trap is loaded by allowing an ion beam with current ${ }_{91} \approx 800 \mathrm{pA}$ to pass through the trap for $100 \mathrm{~s}$ while the longi92 tudinal trap potentials are in loading configuration. After the ${ }_{93}$ loading phase, the trap is closed by raising the potential of ${ }_{94}$ the downstream end cap and the system is left to evolve for ${ }_{95} 10 \mathrm{~s}$ until it reaches equilibrium. This settling time agrees with 96 typical thermalization rates of $\approx 1-5 \mathrm{~s}$ encountered in ion-ion ${ }_{97}$ Coulomb collisions [26, 27]. In this way, reproducible initial 98 conditions for the ensuing experiments are established. Af99 ter the loading process, $1.5-2.0 \times 10^{4}$ anions with an average 100 density of $3-5 \times 10^{4} \mathrm{~cm}^{-3}$ are typically confined in the trap. ${ }_{101}$ The ion cloud is strongly elongated along the axis with a typi$102 \mathrm{cal}$ aspect ratio of 1:40. Most of the anions are $\mathrm{O}^{-}$and a small 103 fraction $(<10 \%)$ is composed of contaminants. These parti104 cles with a mass of 28-39 u could not be clearly identified 105 [25], but remain in the trap over very long times and are un106 affected by the green laser light. These background ions are 107 apparently less prone to recombination with residual gas due 108 to their higher electron affinity.

109 The number $N$ of trapped anions and their temperature $k_{B} T$ 110 are recorded destructively. At the end of each trial, the trap is 111 opened by switching the downstream end cap to the rod po112 tential. The trapped particles are then free to move towards a 113 micro-channel plate (MCP) detector located at a distance of ${ }_{114} \approx 5 \mathrm{~mm}$ from the exit plate of the trap. The MCP is equipped 115 with a phosphor screen that converts the amplified current 116 of secondary electrons into light, which in turn is recorded 117 with a digital camera. The MCP image is an axial projec118 tion of the ejected ion cloud. The radially confining pseu119 dopotential has approximately cylindrical symmetry near the 120 axis, whereas for large excursions the radial potential becomes 121 slightly asymmetrical. The axial confinement can be consid122 ered a simple box potential. Assuming thermal equilibrium, ${ }_{123}$ anions arrange in the trap according to the Boltzmann distri124 bution. Furthermore, in the approximation of a harmonic ra${ }_{125}$ dial potential, the average values of the kinetic and potential
${ }_{126}$ energies are equal (equipartition) [20].

${ }_{127}$ Thus, the radial temperature can be extracted by fitting 128 the density function $p(r) \propto \exp \left(-r^{2} / \sigma^{2}\right)$ to the radial in129 tensity of the images. The spatial width $\sigma$ of the anion ${ }_{130}$ cloud is related to the temperature of the ions by the expres${ }_{131}$ sion $\sigma=\sqrt{k_{B} T /\left(m \omega^{2}\right)}$, where $k_{B}$ is the Boltzmann con${ }_{132}$ stant and $\omega$ is the angular frequency of the anions' radial ${ }_{133}$ oscillations in the trap. The latter can be approximated by ${ }_{134} \omega=\sqrt{2} e V_{p p} /\left(2 m \Omega_{R F} r_{0}^{2}\right)$, where $m$ is the anion mass [28]. 135 The systematic uncertainty of the radial position was esti136 mated by a Monte Carlo simulation to be of the order of $10 \%$. ${ }_{137}$ This uncertainty and that of $V_{p p}$ affect all acquisitions simi138 larly. Therefore, these systematic uncertainties are omitted in 139 all graphs. The axial ejection was not locked to the RF phase, 140 and no dependence of the measured ion temperature on this ${ }_{141}$ (arbitrary) phase was observed.

The laser beam with a Gaussian radial profile is focused 143 into the trap region using a biconvex lens with focal length ${ }_{144} 300 \mathrm{~mm}$ located 294(1) $\mathrm{mm}$ from the focus. The position of 145 the laser beam inside the trap is adjusted by displacing the 146 lens in the plane perpendicular to the light propagation. In a ${ }_{147}$ Cartesian reference frame $(x, y, z)$, where the trap is aligned 148 with the $z$ axis and the laser light propagates in the $y$ direction, 149 the expression for the laser beam intensity $I$ as a function of 150 the $x$ coordinate is thus

$$
I(x)=\frac{I_{0}}{\sqrt{\pi w}} \exp \left(\frac{(x-d)^{2}+z^{2}}{w^{2}}\right),
$$

${ }_{151}$ where $w=0.35(25) \mathrm{mm}$ is the diameter of the beam waist and ${ }_{152} d$ is the displacement of the laser beam in the $x$ direction.

${ }_{153}$ The pressure in the experiment chamber is about $5 \times$ ${ }_{154} 10^{-10}$ mbar. Due to collisions of anions with residual gas we 155 observe both a buffer-gas cooling effect with a final temper156 ature $k_{B} T \approx 0.75 \mathrm{eV}$ and a limited lifetime ( $\left.<2 \mathrm{~min}\right)$. In or${ }_{157}$ der to achieve more efficient cooling, the most energetic an${ }_{158}$ ions should be removed from the trap. Those particles with 159 the highest total energy are also those with the highest radial 160 amplitude at their maximal radial excursion. By adjusting the ${ }_{161}$ displacement $d$ of the laser beam waist from the axis, it is ${ }_{162}$ possible to heat or cool the ion ensemble by selectively inter163 acting with anions orbiting close to or far from the trap axis. ${ }_{164}$ If $d^{*}$ denotes the laser displacement for which neither a net 165 cooling nor a net heating effect is achieved, setting the av166 erage energy of the illuminated anions equal to the total av167 erage energy per anion in the trap we obtain the expression ${ }_{168} d^{*}=\sqrt{\left(w^{2}+\sigma^{2}\right) / 2}$, which will be used to support the inter169 pretation of our experimental results.

We performed three sets of measurements to demonstrate 171 cooling by selective photodetachment. In all cases, the ion 172 evolution with and without the laser radiation interacting with 173 the anions was recorded for the same time duration. In this 174 way, it was possible to directly compare the evolution of the 175 system with and without laser interaction.

176 We first varied the displacement $d$ of the laser beam from 177 the trap axis by adjusting the position of the focusing lens. 


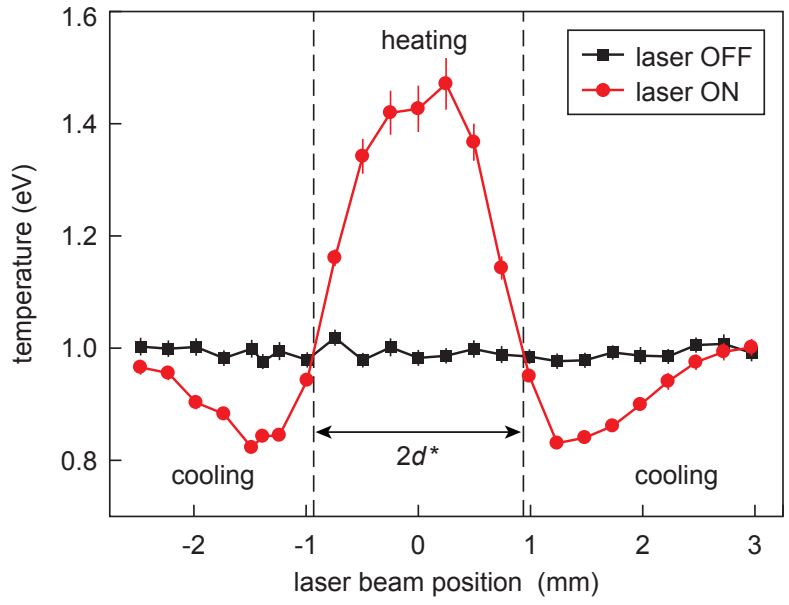

FIG. 2. Final anion temperature as a function of the lens (and hence laser beam) displacement from the trap axis after $12 \mathrm{~s}$ of laser illumination. Vertical dashed lines indicate the lens positions at which neither ion heating nor cooling occurs.

${ }_{178}$ The laser shutter was opened for $12 \mathrm{~s}$ at a laser power of $17911 \mathrm{~mW}$. Figure 2 shows a comparison of the final tempera180 tures obtained with and without illumination. A temperature 182 reduction is observed if ions with large oscillation amplitudes 183 are photodetached, while the ensemble is heated if ions closer 184 to the trap axis are removed. The laser beam displacement $d^{*}$, 185 the position where neither cooling nor heating occurs, corre186 sponds to the cross-over points of the the two data sets shown ${ }_{187}$ in the figure. The value $d_{\mathrm{exp}}^{*}=0.93(5) \mathrm{mm}$ compares well ${ }_{188}$ with the calculated value $d_{\text {theo }}^{*}=1.10(15) \mathrm{mm}$, thus support189 ing the explanation of the cooling mechanism.

190 We then positioned the laser beam to remove anions at a 191 distance $d=1.5 \mathrm{~mm}$ from the trap axis (minima of the red line 192 in Fig. 2, maximum cooling) and measured the storage time as 193 a function of the laser power from $6 \mathrm{~mW}$ to $27 \mathrm{~mW}$. Figure 3 194 shows that, as expected, the lifetime of the anions decreases 196 due to photodetachment. The decrease in the number of ions 197 was modeled with an exponential decay law with a constant 198 fraction $N_{\text {cont }}=1.3 \times 10^{3}$ to account for contaminant ions that 199 resist photodetachment:

$$
N=N_{0} \exp \left(-\frac{t}{\tau}\right)+N_{\text {cont }} .
$$

200 The fit results of the ion lifetimes are indicated in Tab. I.

${ }_{202}$ As mentioned above, the ion temperature of the trapped an${ }_{203}$ ions decreases even without laser irradiation, due to cooling 204 by collisions and thermalization with residual gas. However, 205 Fig. 4 shows that the cooling rate is strongly enhanced by the ${ }_{208}$ detachment laser. The thermalization curve was fitted with the 208 following expression:

$$
T=\left(T_{0}-T_{\infty}\right) \exp \left(-\frac{t}{\theta}\right)+T_{\infty},
$$

209 where $T_{0}$ is the starting temperature, $T_{\infty}$ is the asymptotic final 210 temperature, and $\theta$ is the characteristic cooling time. The pa${ }_{211}$ rameters obtained from the fits are given in Tab. II. The cool-

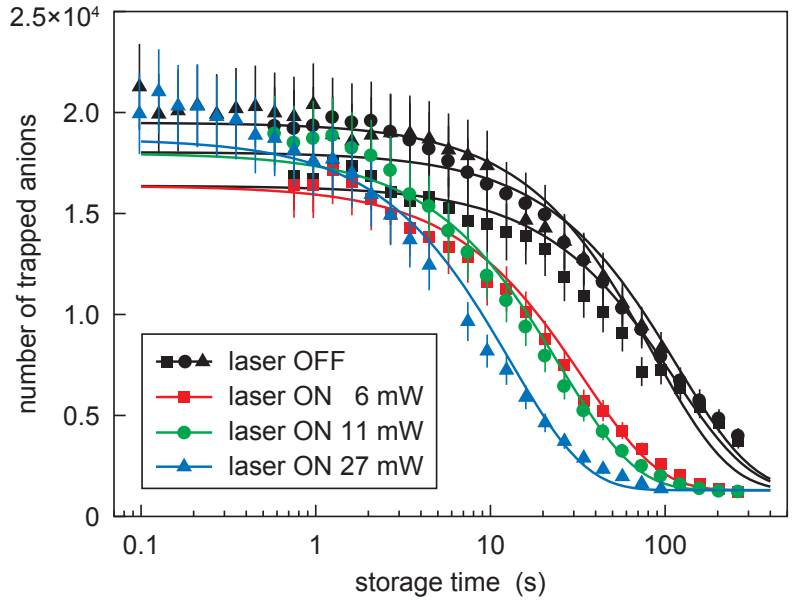

FIG. 3. Number of $\mathrm{O}^{-}$ions in the trap as a function of storage time. The measurements with the laser on were acquired with the laser beam positioned at $d=1.5 \mathrm{~mm}$ (Eq. 1). Data sets with laser on and off denoted with the same symbols were taken contemporaneously under otherwise identical conditions. Solid lines are fits to the data using the function of Eq. 2

TABLE I. Lifetime parameters extracted using Eq. 2 with and without laser illumination. Grouped data sets were taken under similar conditions.

\begin{tabular}{lcc}
\hline \hline Laser power & $N_{0} \times 10^{-4}$ & $\tau(\mathrm{s})$ \\
\hline $\mathrm{OFF}$ & $1.51(5)$ & $113(7)$ \\
$6 \mathrm{~mW}$ & $1.51(4)$ & $34(1)$ \\
\hline $\mathrm{OFF}$ & $1.67(4)$ & $117(6)$ \\
$11 \mathrm{~mW}$ & $1.67(4)$ & $25.1(9)$ \\
\hline $\mathrm{OFF}$ & $1.82(2)$ & $86(4)$ \\
$27 \mathrm{~mW}$ & $1.74(5)$ & $13.0(6)$ \\
\hline \hline
\end{tabular}

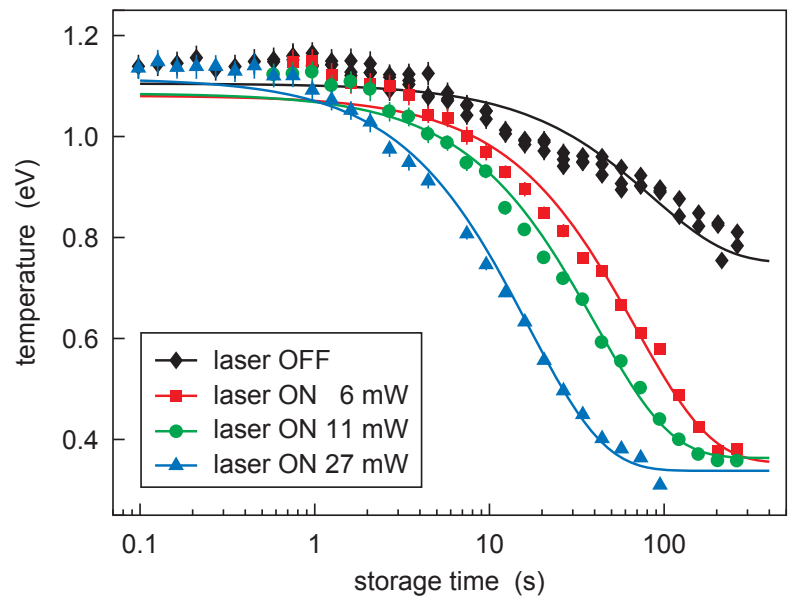

FIG. 4. Temperature of $\mathrm{O}^{-}$anions in the trap as a function of storage time. The measurements with the laser were acquired with $d=1.5 \mathrm{~mm}$ (Eq. 1). Solid lines are fits to the data using the function of Eq. 3. 
TABLE II. Starting temperature, asymptotic final temperature and cooling time obtained from the fit of Eq. 2 to the data of Fig. 4.

\begin{tabular}{llcc}
\hline \hline Laser power & $T_{0}(\mathrm{eV})$ & $T_{\infty}(\mathrm{eV})$ & $\theta(\mathrm{s})$ \\
\hline OFF & $1.105(7)$ & $0.75^{\mathrm{a}}$ & $85(5)$ \\
$6 \mathrm{~mW}$ & $1.08(2)$ & $0.35(1)$ & $69(4)$ \\
$11 \mathrm{~mW}$ & $1.09(1)$ & $0.363(7)$ & $40(2)$ \\
$27 \mathrm{~mW}$ & $1.12(2)$ & $0.338(7)$ & $16.8(7)$ \\
\hline \hline
\end{tabular}

a Parameter obtained from fit of Fig. 5 and held fixed for this fit

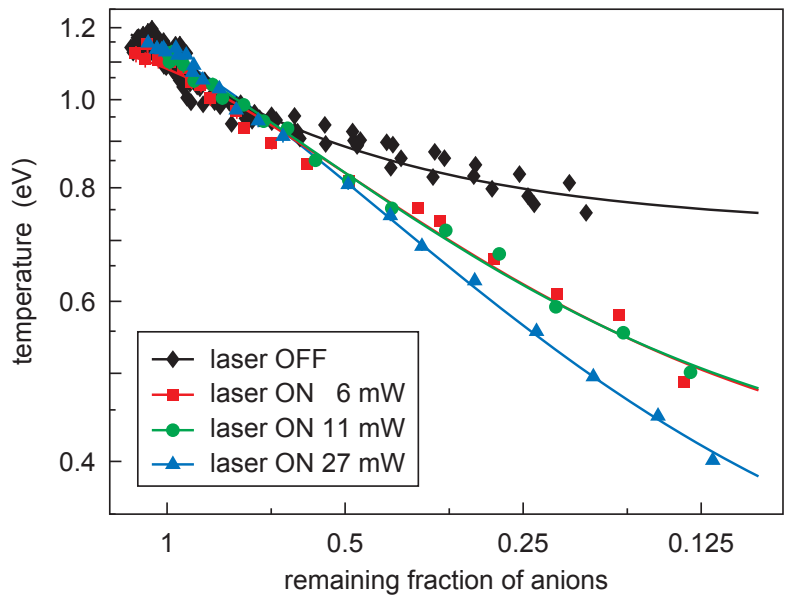

FIG. 5. Anion temperature as a function of the fraction of ions remaining in the trap. The number of remaining anions is normalized to the initial population before cooling. Error bars are comparable to the size of the data points. Solid lines are second-order polynomial fits and are meant to guide the eye.

212

${ }_{213}$ ing rate $\theta$ is enhanced in the presence of the laser light, and

214 more so for higher laser powers. Most importantly, the asymp-

215 totic final temperature $T_{\infty}$ is also significantly lower with the

${ }_{216}$ laser on $(\approx 0.35 \mathrm{eV})$ than without laser $(\approx 0.75 \mathrm{eV})$.

217 Adopting this cooling scheme, it was possible to prepare a

${ }_{218}$ larger ensemble of anions at a lower temperature than without

${ }_{219}$ laser excitation. To highlight this effect, Fig. 5 shows the ion

220 temperature as a function of the fraction of anions remaining

${ }_{222}$ in the trap after cooling. For instance, when $3 / 4$ of the an-

${ }_{223}$ ions have been removed or lost, a temperature of $0.56(17) \mathrm{eV}$

224 is obtained by cooling at $27 \mathrm{~mW}$ laser power, compared to

$2250.80(24) \mathrm{eV}$ with the laser off. To reach this condition the

226 laser interacted with the ions for $<20 \mathrm{~s}$, whereas a similar ion

${ }_{227}$ loss took more than 10 times longer without laser due to the

228 lower loss rate with only collisional detachment.

229 Finally, we monitored the temperature of the stored anions 260

230 after a brief laser-induced cooling cycle. The system was pre- 260

${ }_{231}$ pared in a similar condition as previously, with the laser beam 207 ${ }_{232}$ position fixed at $d=1.5 \mathrm{~mm}$. After selective photodetach- ${ }_{268}$ the ion cloud in the axial direction to enhance overlap and ${ }_{233}$ ment during $12 \mathrm{~s}$ at a laser power of $11 \mathrm{~mW}$, the ion cloud ${ }_{269}$ ion-ion collision rate. Displacing the laser position radially ${ }_{234}$ was allowed to evolve freely in the trap. The result is shown 270 inward during cooling could help optimize the cooling rate 235 in Fig. 6, where the ion temperature is plotted as a function of 271 and decrease ion losses. Finally, the cooling scheme could be

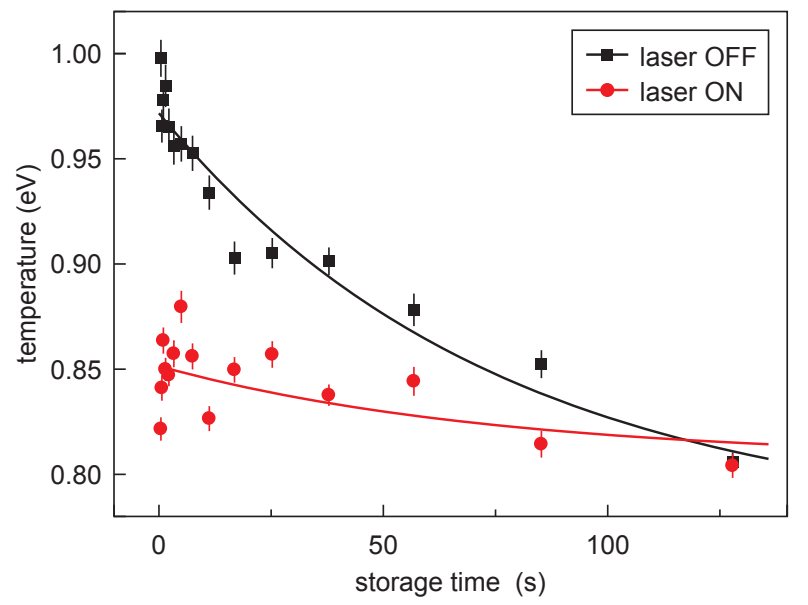

FIG. 6. Anion temperature as a function of storage time after the end of laser-induced cooling. Solid lines are simple exponential fits to the data.

${ }_{238}$ the additional storage time after cooling. The figure shows that 238 the temperature reduction induced by the selective laser pho239 todetachment persists quasi indefinitely. This indicates that af240 ter evaporative cooling the entire anion cloud reaches thermal ${ }_{241}$ equilibrium, as expected. While the data show that a similar 242 final temperature is also reached without selective photode243 tachment (at this low laser power), it is evident from Fig. 3 244 that the remaining fraction of ions is much smaller after $120 \mathrm{~s}$ 245 of storage time without laser $(\approx 25 \%)$ than after $12 \mathrm{~s}$ of cool246 ing $(\approx 75 \%)$.

247 In summary, we have presented the first cooling of trapped ${ }_{248}$ negative ions induced by a light field. Laser light is used to 249 selectively remove the hottest particles by photodetachment. 250 The selection is applied by geometrically addressing those an251 ions with the largest radial oscillation amplitude. In this way, ${ }_{252}$ the ions' temperature was reduced from $1.15 \mathrm{eV}(13,000 \mathrm{~K})$ to ${ }_{253} 0.33 \mathrm{eV}(3800 \mathrm{~K})$ within about $100 \mathrm{~s}$. With this technique the 254 stored anions can be cooled faster and to lower temperatures 255 than can be achieved by buffer gas cooling with residual gas. 256 For instance, we observed a more than twofold improvement 257 in the limiting final temperature, and that temperature can be 258 reached about 10 times faster than by buffer gas cooling alone. The technique can easily be extended to other negative ion so species, assuming a laser is available that can photodetach ${ }_{261}$ them directly. State-of-the-art lasers for this task are gener262 ally available, as typical electron affinities are of the order of ${ }_{263} 1 \mathrm{eV}$. Heavier anions up to ${ }^{139} \mathrm{La}$ can be confined in our Paul 264 trap with an RF drive amplitude of 1.9-2.0 kV (peak-to-peak), 265 or at a correspondingly lower amplitude in a trap with smaller 66 radial dimensions. Faster cooling can be realized by illumi87 nating ions over a broader axial region and by compressing inward during cooling could help optimize the cooling rate
and decrease ion losses. Finally, the cooling scheme could be 
272 combined with an additional resonant excitation step, which 307 273 would allow the laser to be red-detuned for Doppler selection 308 274 of the hottest particles. This would enhance both the selectiv- ${ }^{309}$ 275 ity and the efficiency of the overall cooling process.

${ }_{276}$ The authors thank the MPIK mechanical workshop for their 277 excellent work in constructing the linear RF trap. This work 278 was supported by the European Research Council (ERC) un279 der Grant No. 259209 (UNIC).

304 [10] A. Kellerbauer and J. Walz, New J. Phys. 8, 45 (2006).

305 [11] A. Kellerbauer et al. (AEGIS Proto-Collaboration), Nucl. In-
* Corresponding author; a.kellerbauer@ cern.ch

] J.-S. Chen, S. M. Brewer, C. W. Chou, D. J. Wineland, D. R. Leibrandt, and D. B. Hume, Phys. Rev. Lett. 118, 053002 (2017).

[2] W. Rosenfeld, D. Burchardt, R. Garthoff, K. Redeker, N. Ortegel, M. Rau, and H. Weinfurter, Phys. Rev. Lett. 119, 010402 (2017).

[3] N. Friis, O. Marty, C. Maier, C. Hempel, M. Holzäpfel, P. Jurcevic, M. B. Plenio, M. Huber, C. Roos, R. Blatt, et al., Phys. Rev. X 8, 021012 (2018).

[4] M. H. Anderson, J. R. Ensher, M. R. Matthews, C. E. Wieman, and E. A. Cornell, Science 269, 198 (1995).

[5] K. B. Davis, M.-O. Mewes, M. R. Andrews, N. J. van Druten, D. S. Durfee, D. M. Kurn, and W. Ketterle, Phys. Rev. Lett. 75, 3969 (1995).

[6] M. Drewsen, Physica B 460, 105 (2015).

[7] L. Schmöger, O. O. Versolato, M. Schwarz, M. Kohnen, ${ }^{3} 6$ A. Windberger, B. Piest, S. Feuchtenbeiner, J. Pedregosa- ${ }^{337}$ Gutierrez, T. Leopold, P. Micke, et al., Science 347, 1233 зз8 (2015).

8] W. G. Rellergert, S. T. Sullivan, S. J. Schowalter, S. Ko- 340 tochigova, K. Chen, and E. R. Hudson, Nature 495 (2013). 341

[9] B. Höltkemeier, P. Weckesser, H. López-Carrera, and M. Wei- 342 demuiller, Phys. Rev. Lett. 116, 233003 (2016). strum. Methods B 266, 351 (2008).
[12] U. Warring, M. Amoretti, C. Canali, A. Fischer, R. Heyne, J. O. Meier, C. Morhard, and A. Kellerbauer, Phys. Rev. Lett. 102, 043001 (2009).

[13] C. W. Walter, N. D. Gibson, Y.-G. Li, D. J. Matyas, R. M. Alton, S. E. Lou, R. L. Field, D. Hanstorp, L. Pan, and D. R. Beck, Phys. Rev. A 84, 032514 (2011).

[14] P. Yzombard, M. Hamamda, S. Gerber, M. Doser, and D. Comparat, Phys. Rev. Lett. 114, 213001 (2015).

5 [15] S. Gerber, J. Fesel, M. Doser, and D. Comparat, New J. Phys. 20, 023024 (2018).

[16] S. M. O’Malley and D. R. Beck, Phys. Rev. A 81, 032503 (2010).

[17] C. W. Walter, N. D. Gibson, D. J. Matyas, C. Crocker, K. A. Dungan, B. R. Matola, and J. Rohlén, Phys. Rev. Lett. 113, 063001 (2014).

[18] E. Jordan, G. Cerchiari, S. Fritzsche, and A. Kellerbauer, Phys. Rev. Lett. 115, 113001 (2015).

[19] G. Cerchiari, A. Kellerbauer, M. S. Safronova, U. I. Safronova, and P. Yzombard, Phys. Rev. Lett. 120, 133205 (2018).

[20] A. Crubellier, J. Phys. B 23, 3585 (1990).

7 [21] W. Ketterle and N. J. Van Druten, Adv. At. Mol. Opt. Phys. 37, 181 (1996).

[22] B. M. Penetrante, J. N. Bardsley, M. A. Levine, D. A. Knapp, and R. E. Marrs, Phys. Rev. A 43, 4873 (1991).

[23] W. Chaibi, R. J. Pelaez, C. Blondel, C. Drag, and C. Delsart, Eur. Phys. J. D 58, 29 (2010).

[24] E. Tiesinga, P. J. Mohr, D. B. Newell, and B. N. Taylor, The 2018 CODATA recommended values of the fundamental physical constants (web version 8.0) (National Institute of Standards and Technology, Gaithersburg, 2019), URL http: //physics . nist.gov/constants.

[25] G. Cerchiari, S. Erlewein, P. Yzombard, M. Zimmermann, and A. Kellerbauer, J. Phys. B 52, 155003 (2019).

[26] J. Huba, NRL Plasma Formulary (Naval Research Laboratory, Plasma Physics Division, 2018), URL https://www . nrl .navy. mil/ppd/sites/www . nrl. navy.mil.ppd/files/pdfs/NRL_FORMULARY_18.pdf.

4 [27] L. Spitzer, Physics of Fully Ionized Gases: Second Revised Edition (Dover, 2013), ISBN 9780486449821.

346 [28] M. Drewsen and A. Brøner, Phys. Rev. A 62, 045401 (2000). 